\section{Systemische Therapie bei Gehirnmetastasen}

D as Thema Gehirnmetastasen bildete einen Schwerpunkt auf dem diesjährigen ASCO-Kongress; etwa ein Viertel aller Melanompatienten im Stadium IV haben Gehirnmetastasen („melanoma brain metastases“, MBM) auf. Dr. Andrea Forschner, Tübingen, referierte drei auf dem ASCO präsentierte PhaseII-Studien.

In der COMBI-MB-Studie wurden $\mathrm{Pa}$ tienten mit $\mathrm{BRAF}^{\mathrm{V} 600}$-Mutationen mit Dabrafenib plus Trametinib behandelt [Davies M et al. J Clin Oncol 2017; 35 (Suppl): Abstr 9506]. Der primäre Endpunkt - die intrakraniale Ansprechrate in der Kohorte mit asymptomatischen Patienten ohne vorherige Lokaltherapie - wurde mit $58 \%$ erreicht. Das mediane progressionsfreie Überleben lag bei 5,6 Monaten, das Gesamtüberleben bei 10,8 Monaten.

Die ABC-Studie untersuchte den Effekt von Nivolumab alleine sowie Nivolumab plus Ipilimumab [Long GV et al. J Clin Oncol 2017; 35 (Suppl): Abstr 9508]. Bei asymptomatischen MBMPatienten erwies sich insbesondere die Kombination als intrakranial aktiv. Die intrakraniale Ansprechrate betrug 42\%, das 6-monatige progressionsfreie Überleben $46 \%$ im Vergleich zu $20 \%$ und $29 \%$ unter Nivolumab alleine.

Die Effizienz von Nivolumab plus Ipilimumab analysierte auch die Studie CheckMate 204 [Tawbi HAH et al. J Clin Oncol 2017; 35 (Suppl): Abstr 9507]. Viele Patienten sprachen schon früh an - im Median nach 2,8 Monaten - bei einer intrakranialen Ansprechrate von $59 \%$. Das mediane intrakraniale progressionsfreie Überleben wurde noch nicht erreicht.

Aufgrund dieser Ergebnisse erscheint die systemische Erstlinientherapie eine wichtige Option bei asymptomatischen MBM-Patienten. Offen ist bisher, welche Therapie dabei zuerst verabreicht werden sollte und wie die systemische mit der lokalen Therapie zu kombinieren ist. Dr.Marion Hofmann-Aßmus

Symposium VI: „Brain metastasis: still a dilemma!"

\title{
Metastasiertes uveales Melanom: kaum Erfolge
}

$D^{2}$ as seltene uveale Melanom geht von Melanozyten der Augenhaut (Uvea) aus. Die Prognose ist eher schlecht, die 5-Jahres-Überlebensrate liegt bei etwa $40 \%$. Lokalisierte Tumoren werden operiert oder bestrahlt. Wie Prof. Bastian Schilling, Würzburg, berichtete, sind die Therapieoptionen für das metastasierte uveale Melanom (MUM) nicht so eindeutig. Die Metastasen finden sich meist in der Leber - wobei deren Behandlung das Gesamtüberleben (OS) nicht verbessert.

In der Phase-II-Studie CheckMate 172 wurden MUM-Patienten mit Nivolumab behandelt [Schadendorf D et al. J Clin Oncol 2017; 35 (Suppl): Abstr 9524]. Die Gesamtansprechrate (ORR) lag bei nur $6 \%$, das mediane OS bei elf Monaten. Vergleichbar magere Ergebnisse brachte die Kombination aus Nivolumab plus Ipilimumab [Hogg D et al. J Clin Oncol
2017; 35 (Suppl): Abstr 9554]. „Diese Daten verweisen auf eine sehr limitierte Wirkung von Checkpointinhibitoren bei metastasiertem Aderhautmelanom“, konstatierte Schilling.

Eine zielgerichtete Therapie mit Sorafenib untersuchte die STREAM-Studie mit MUM-Patienten mit stabiler Erkrankung [Scheulen ME et al. J Clin Oncol 2017; 35 (Suppl): Abstr 9511]. Unter Sorafenib erreichten die Patienten ein PFS von 5,5 Monaten versus 1,9 Monaten mit Placebo ( $\mathrm{p}=0,0079)$, die Risikoreduktion betrug etwa $50 \%$, das OS lag bei 14,8 Monaten. „Während Sorafenib bei kutanem Melanom nicht hilft, scheint es beim MUM einen Effekt zu haben“, erklärte Schilling. Dr. Marion Hofmann-Aßmus

Symposium VI: „Metastatic ocular melanoma: an untreatable disease?"

\section{T-Vec in Kombination erhöht die Ansprechraten}

$T^{a}$ alimogen laherparepvec $(\mathrm{T}-\mathrm{Vec})$ ist ein abgeschwächtes Herpes-simplex-1Virus, dessen Erbgut biotechnisch so verändert wurde, dass es Melanomzellen infizieren und sich darin vermehren kann. Einerseits können die Viren die Melanomzellen direkt abtöten, andererseits werden Killerzellen angelockt, die infizierte Zellen zerstören. Über die Möglichkeit, dieses onkolytische Wirkprinzip mit anderen immuntherapeutischen Wirkmechanismen zu kombinieren, berichtete Olivier Michielin, Lausanne/Schweiz.

Ergebnisse einer Open-Label-Studie der Phase II zeigen, dass eine solche Kombination die Durchschlagskraft erhöhen kann. 98 Patienten mit nicht resezierten Melanomen in den Stadien IIIIV hatten dafür T-Vec zusammen mit Ipilimumab erhalten, das über eine Blockade von CTLA-4 die Arbeit von zytotoxischen T-Zellen unterstützt. 100 Kontrollpatienten hatten nur Ipilimumab erhalten. Die Ansprechrate nach sechs Monaten in der Kombinationsgruppe lag bei $38,8 \%$, verglichen mit $18,0 \%$ bei den Kontrollen. Die Rate an Komplettremissionen erreichte $13,3 \%$ versus 7,0\%. Progressionsfrei überlebten die Probanden im Median 8,2 versus 6,4 Monate, wobei die Therapieresponse allerdings bei $89 \%$ der Patienten im Kombinationsarm und bei $83 \%$ der Patienten unter IpilimumabMonotherapie erhalten blieb.

Auch erste Ergebnisse einer Kombination von T-Vec mit dem Immuncheckpointinhibitor Pembrolizumab lassen aufhorchen. An der Phase Ib der MASTERKEY-265-Studie waren 21 Patienten mit Melanomen im Stadium IIIB-IV beteiligt, die Ansprechrate lag bei rund $57 \%$ und die Rate von Komplettremissionen erreichte rund $24 \%$.

Derzeit werden laut Michielin $660 \mathrm{~Pa}$ tienten für die Phase III von MASTERKEY-265 rekrutiert, die randomisiert entweder Pembrolizumab plus Placebo oder Pembrolizumab und T-Vec erhalten sollen. Beteiligt sind weltweit 128 Forschungszentren, darunter zwölf deutsche. Hier geht es dann in den primären Endpunkten auch um das progressionsfreie sowie das Gesamtüberleben. Als Termin für das Studienende ist September 2022 anvisiert, die Daten für die primären Endpunkte sollen Ende 2018 vorliegen.

Dr. Robert Bublak

Parallel Session I: „Intralesional treatments: current developments. T-VEC alone or in combination" 